Editorial

\title{
COVID-19 (Coronavirus Disease-19): Traditional Chinese Medicine including Acupuncture for Alleviation - A Report from Wuhan, Hubei Province in China
}

\author{
Fengxia Liang ${ }^{1,2,+}$, Gerhard Litscher ${ }^{1,2,+, *}$
}

1. Hubei University of Chinese Medicine, Institute of Acupuncture \& Moxibustion, Wuhan, 430061 China; E-Mails: fxliang5@hotmail.com; gerhard.litscher@medunigraz.at

2. Research Unit of Biomedical Engineering in Anesthesia and Intensive Care Medicine, Research Unit for Complementary and Integrative Laser Medicine, and Traditional Chinese Medicine (TCM) Research Center Graz, Medical University of Graz, 8036 Graz, Austria

† These authors contributed equally to this work.

* Correspondence: Gerhard Litscher; E-Mail: gerhard.litscher@medunigraz.at

Special Issue: Modernization of Acupuncture Education and Research

OBM Integrative and Complementary Medicine

2020, volume 5 , issue 1

doi:10.21926/obm.icm.2001009
Received: February 25, 2020

Accepted: February 25, 2020

Published: February 26, 2020

\begin{abstract}
Traditional Chinese Medicine (TCM) has been used to prevent and alleviate epidemic diseases for hundreds of years. The combination of TCM and Western medicine can effectively reduce fever; alleviate cough, fatigue, diarrhoea and other symptoms of patients with mild COVID-19 (Coronavirus disease-19). For the treatment of moderate and severe syndromes, the integrative approach can reduce lung exudation and inhibit the further development of the disease. However the risk of infection is a limit for example in needle acupuncture treatment. Computer-controlled laser acupuncture and/or robot-controlled acupuncture are nowadays not far away from clinical use.
\end{abstract}

\section{Keywords}

COVID-19 (Coronavirus disease); Novel Coronavirus Pneumonia (NCP); Traditional Chinese Medicine (TCM); acupuncture; laser acupuncture; robot-controlled acupuncture

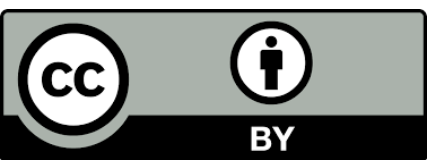

(C) 2020 by the author. This is an open access article distributed under the conditions of the Creative Commons by Attribution License, which permits unrestricted use, distribution, and reproduction in any medium or format, provided the original work is correctly cited. 


\section{Introduction}

One important thing in advance: With acupuncture you will not be able to cure coronavirus disease (COVID-19). However, it seems appropriate to report on different strategies in which symptoms can be alleviated, as is currently (February 25, 2020) practiced in Wuhan in Hubei Province in China.

"Coronavirus infections in China continue to swell by thousands a day, prompting epidemiologists to estimate when the outbreak will peak. Some suggest the climax, when the number of new infections in a single day reaches its highest point, will happen any time now. Others say that it is months away and that the virus will infect millions - or in one estimate hundreds of millions - of people first."[1].

Traditional Chinese Medicine (TCM) has been used to prevent and alleviate epidemic diseases for hundreds of years. Smallpox prevention in China was an epoch-making initiative in the history of human preventive medicine. Several editions of 'The Diagnosis and Treatment Plan for Novel Coronavirus Pneumonia (NCP)' (COVID-19) have been issued by the National Health Committee of China. The plan fully attaches importance to the diagnosis and treatment of integrated Traditional Chinese and Western medicine, effectively guiding clinical treatment work, especially emphasizing the role of Chinese medicine, and establishing a consultation system of integrated Traditional Chinese and Western medicine. All over the country, effective treatment of COVID-19 with integrated Chinese and Western medicine has been carried out. There are also specialized scientific research institutions, hospitals and enterprises to jointly carry out online consultation of TCM experts for COVID-19 patients [2, 3].

\section{Application of TCM}

The 'National Plan' guides the clinical practice of TCM treatment as early as possible, reducing fever, improving cough, asthma and gastrointestinal symptoms in time and effectively, and improving the cure rate. The combination of TCM and Western medicine can effectively alleviate the fever, cough, fatigue, diarrhoea and other symptoms of patients with mild NCP. For the treatment of moderate and severe patients, the integrative treatment can reduce lung exudation and inhibit the further development of the disease.

\section{Application of Acupuncture and Moxibustion}

Acupuncture and moxibustion are important parts of TCM. Historically, burning moxa leaves and moxibustion on acupoints (purulent moxibustion) have been used to prevent epidemics. Moxibustion, through the stimulation of warming acupoints, has the functions of warming yang, dispersing cold, activating meridians, strengthening yang and removing toxin, etc. Modern research on moxibustion has disclosed its obvious effect in regulating immune function and autonomic nervous system [4].

For further COVID-19 prevention, diagnosis and medical treatment, China Association of Acupuncture-Moxibustion (CAAM), as a group member of the World Federation of Acupuncture - 
Moxibustion Societies (WFAS) has organized acupuncture and moxibustion experts to work immediately, and has formed a group of experts for the prevention and control of the COVID-19. The group has formulated and published Guidelines on Acupuncture and Moxibustion Intervention for Novel Coronavirus Pneumonia (first edition).

Although some research showed that there are antiviral components in moxa, the influence of smoke will be considered when it is used in the closed environment of the isolation ward, so acupuncture point application, pressing pills on ear acupuncture points, acupoint pressing and massage, etc. are more widely used in the practice. The commonly recommended acupoints for moxibustion and acupoint application are Zusanli (ST36), Guanyuan (CV4), Dazhui (GV14), Fengmen (BL12) and Feishu (BL13), other acupoints such as Zhongwan (CV12) and Shenque (CV8) are applied according to the symptoms. The acupoint therapy improves the symptoms such as poor appetite, coughing, insomnia, headache of the COVID-19 patients effectively [5].

\section{Application of Qigong}

China has built up to now (February 25, 2020) more than a dozen of temporary shelter hospitals in Wuhan to treat the patients with mild COVID-19. Some of the patients exercise Qigong under the guidance of doctors [6]. Qigong can improve the pain situation throughout and fatigue of the COVID-19 patients.

\section{New Technologies in Acupuncture Research}

Although TCM can be used sensibly for COVID-19 [7], traditional needle acupuncture has some limitations. In this special situation, there is the risk of infection as well as practical handling. There are already alternatives for both. Computer-controlled laser acupuncture is one of the possibilities [8] and robot-controlled acupuncture [9] is nowadays not far from clinical use. This vision of "robot-controlled acupuncture" is already a reality [9] as one of the authors (G.L.) has been reported on the 'Binhu lecture' in Wuhan on September 19, 2019 [10].

\section{Acknowledgments}

The article has been drafted as cooperative report in Wuhan and additional parts and current literature have been added from the author from Graz. Professor Fengxia Liang is Director of the Institute of Acupuncture and Moxibustion of the Hubei University of Chinese Medicine in Wuhan, China. She has been Visiting Scientist at the Medical University of Graz in Austria in 2017. Professor Gerhard Litscher is also Visiting and Honorary Professor at Hubei University of Chinese Medicine in Wuhan, China and at Hubei Provincial Collaborative Innovation Center of Preventive Treatment by Acupuncture \& Moxibustion, Wuhan, China, respectively.

\section{Funding}

There was no funding for this article.

\section{Competing Interests}

The authors have declared that no competing interests exist. 


\section{References}

1. Cyranoski D. When will the coronavirus outbreak peak? Nature News. 2020 February 18. Doi: 10.1038/d41586-020-00361-5

2. Stop the Wuhan virus. Nature. 2020; 577: 450. Doi: 10.1038/d41586-020-00153-x

3. Li M, Yang X, Li K, Xie YQ. Traditional Chinese Medicine for novel coronavirus pneumonia treatment: main force or supplement? TMR Integrative Medicine, 2020; 1-3. Doi: 10.12032/TMR20200204158.

4. Shu Q, Wang H, Litscher D, Wu S, Chen L, Gaischek I, et al. Acupuncture and moxibustion have different effects on fatigue by regulating the autonomic nervous system: A pilot controlled clinical trial. Sci Rep. 2016; 6: 37846. Doi: 10.1038/srep37846.

5. Lu P, Wang S, Tang Z. Feasibility analysis of early intervention of moxibustion in the prevention and treatment of novel coronavirus pneumonia. Acta Chinese Med Pharm. 2020; 19: 1-12.

6. Liang FX, Huang $Q$, Chen $R, W u S, L u ~ G$, Liu J, et al. Healthcare-related qigong for the prevention of lifestyle-related diseases. OBM Integrat Complement Med. 2017; 2: 002; doi:10.21926/obm.icm.1702002.

7. Luo Y, Wang CZ, Hesse-Fong J, Lin JG, Yuan CS. Application of Chinese medicine in acute and critical medical conditions. Am J Chinese Med. 2019; 47: 1223-1235. https://doi.org/10.1142/S0192415X19500629

8. Litscher $G$, Litscher D. Scientific aspects of innovative laser medicine. In: Bahr F, Litscher $G$ (eds) Laser acupuncture and innovative laser medicine. Munich, Germany: Bahr\&Fuechtenbusch; 2018. p. 13-77.

9. Lan KC, Litscher G. Robot-controlled acupuncture - an innovative step towards modernization of the ancient traditional medical treatment method. Medicines. 2019; 6: 87. https://doi.org/10.3390/medicines6030087

10. Litscher G. From tradition to future of acupuncture - high-tech acupuncture, laser acupuncture, automated pulse diagnosis, robot-controlled acupuncture. Li Binhu (Li Shizhen). Lecture at Hubei University of Chinese Medicine; 2019 Sep 19; Wuhan, China.

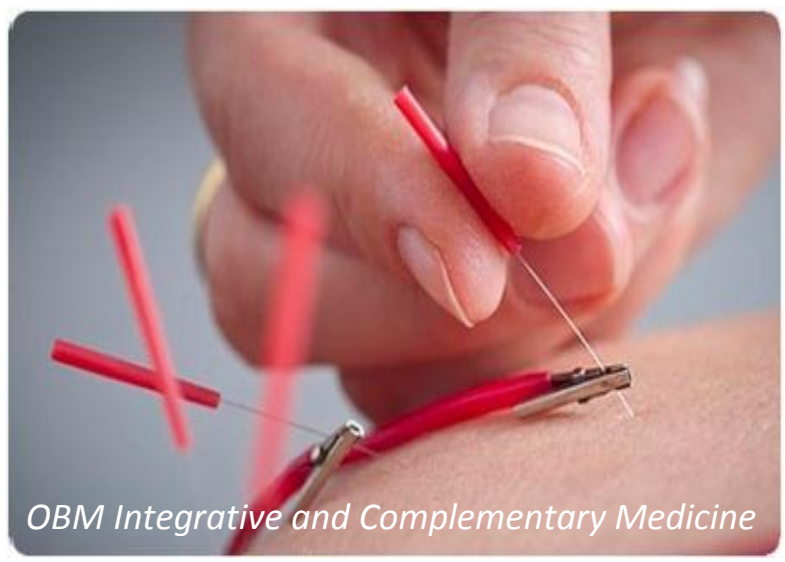

Enjoy OBM Integrative and Complementary Medicine by:

1. Submitting a manuscript

2. Joining in volunteer reviewer bank

3. Joining Editorial Board

4. Guest editing a special issue

For more details, please visit: http://www.lidsen.com/journals/icm 